\title{
Classification of different pineapple varieties grown in Malaysia based on volatile fingerprinting and sensory analysis
}

\author{
Ola Lasekan * ${ }^{*}$ and Fatma Khalifa Hussein
}

\begin{abstract}
Background: Pineapple is highly relished for its attractive sweet flavour and it is widely consumed in both fresh and canned forms. Pineapple flavour is a blend of a number of volatile and non-volatile compounds that are present in small amounts and in complex mixtures. The aroma compounds composition may be used for purposes of quality control as well as for authentication and classification of pineapple varieties.

Results: The key volatile compounds and aroma profile of six pineapple varieties grown in Malaysia were investigated by gas chromatography-olfactometry (GC-O), gas-chromatography-mass spectrometry and qualitative descriptive sensory analysis. A total of 59 compounds were determined by GC-O and aroma extract dilution analysis. Among these compounds, methyl-2-methylbutanoate, methyl hexanoate, methyl-3-(methylthiol)-propanoate, methyl octanoate, 2,5-dimethyl-4-methoxy-3(2H)-furanone, $\delta$-octalactone, 2-methoxy-4-vinyl phenol, and $\delta$-undecalactone contributed greatly to the aroma quality of the pineapple varieties, due to their high flavour dilution factor. The aroma of the pineapples was described by seven sensory terms as sweet, floral, fruity, fresh, green, woody and apple-like.

Conclusion: Inter-relationship between the aroma-active compounds and the pineapples revealed that 'Moris' and 'MD2' covaried majorly with the fruity esters, and the other varieties correlated with lesser numbers of the fruity esters. Hierarchical cluster analysis (HCA) was used to establish similarities among the pineapples and the results revealed three main groups of pineapples.
\end{abstract}

Keywords: Pineapple varieties, Volatile fingerprinting, PCA, HCA, Sensory evaluation, GC-O

\section{Background}

Pineapple (Ananas comosus L. Merr) which is one of the most popular exotic fruits in the world trade is widely distributed in tropical regions such as the Philippines, Thailand, Malaysia and Indonesia. In 2016, the global pineapple production was estimated at 24.78 million metric tons with Costa Rica (2930.66 metric tons), Brazil (2694.56 metric tons), Philippines (2612.47 metric tons), India (1964 metric tons),Thailand (1811.59 metric tons, and Nigeria (1591.28 metric tons) as the top five pineapple producers in the world [1]. Other important producers are: Indonesia, China, India, Mexico, and Colombia

*Correspondence: olaniny56@gmail.com

Department of Food Technology, University Putra Malaysia, 43400 UPM Serdang, Malaysia
[2]. Malaysia is part of a new group of pineapple-producing countries. Malaysia exported approximately 20,000 tons of fresh pineapples annually [2]. The main pineapple varieties grown in Malaysia are: 'Moris', 'N36, 'Sarawak', 'Gandul, 'Yankee,' 'Josapine,' 'Maspine,' and most recently 'MD2'. Some of these varieties such as N36 and Josapine were locally developed for the local fresh fruit market.

Pineapple is highly relished for its attractive sweet flavour and it is widely consumed in both fresh and canned forms [3]. Pineapple flavour is a blend of a number of volatile and non-volatile compounds that are present in small amounts and in complex mixtures [4]. The volatile constituents of pineapples have been studied extensively and more than 280 compounds have been reported $[4,5]$. Aroma chemicals are organic compounds with defined chemical structures. They are generated by organic or 
bio-catalytic synthesis or isolated from microbial fermentations [4]. There are many pathways involved in volatile biosynthesis starting from lipids [6], amino acids [7], terpenoids [8] and carotenoids [9]. Once the basic skeletons are produced via these pathways, the diversity of volatiles is achieved via additional modification reactions such as acylation, methylation, oxidation/reduction and cyclic ring closure [6]. As the content of aroma compounds in pineapple depends on many factors such as the climatic and geographical origin [10], varieties [11], different stages of ripening [12], and postharvest storage conditions [13], the aroma compounds composition may be used for purposes of quality control as well as for authentication and classification of pineapple varieties.

Fingerprinting techniques, based on chemical composition and multivariate statistical analysis have been used in characterising or classifying wines according to origin, quality, variety and type $[14,15]$. It was also used in the authentication of green-ripe sea-freighted and airfreighted pineapple fruits harvested at full maturity [16]. Application of untargeted fingerprinting techniques as a means of gaining insight into the reaction complexity of a food system has received tremendous interest among researchers [17]. Fingerprinting is defined as a more unbiased and hypothesis-free methodology that considers as many compounds as possible in a particular food fraction [18]. Fingerprinting doesn't concentrate on a specifically known compound, rather it allows for an initial fast screening to detect differences among samples. Meanwhile, chemometric techniques such as principal component analysis (PCA) and hierarchical cluster analysis (HCA) are employed in the analysis of generated data. PCA is often complemented with HCA to explore data sets obtained by gas chromatography. This method has been used in the classification of wines based on their volatile profiles [19]. Multivariate techniques of data analysis represent a useful statistical tool to differentiate between different fruit varieties [20]. Also, this chemometric approach has been used to classify muskmelon [21], tomato fruit [22], and citrus juice [20].

Although much work has been done on volatile fingerprinting in apple fruits [23], and grape fruits [24], there has been no systematic study on volatile fingerprinting of fresh pineapple fruits grown in Malaysia. The purpose of this study were: (1) to identify and quantify the volatile compounds in six different varieties of pineapples grown in Malaysia (Moris, Maspine, MD2, N36, Josapine and Sarawak) and (2) apply fingerprinting technique to determine which volatile compounds may be potential markers for pineapple varieties grown in Malaysia.

\section{Results and discussion}

\section{Sensory evaluation}

The aroma qualities of the six different pineapple varieties were elucidated by ten trained panellists. The obtained relative standard deviation from the mean aroma quality intensities varied within the range of $1.2-5.9 \%$ depending on the pineapple variety and the aroma quality. The details of the aroma qualities of the pineapples are listed in Table 1. Results of the aroma qualities revealed significant differences $(\mathrm{p}<0.05)$ among varieties for all attributes. For instance, while pineapple 'MD2' presented the highest intensities for sweetness (8.62), floral (6.88) and apple-like (8.31) attributes, 'Moris' produced the highest intensities for fruity (6.83) and fresh (7.31) attributes, respectively. On the other hand, 'Sarawak' had the strongest woody (7.46) and green (7.62) attributes. The other pineapple varieties ('Josapine', 'N36' and 'Maspine') produced varied aroma responses. 'Josapine' had strong sweet and woody attributes with relatively low floral aroma. 'Maspine' exhibited strong sweet and green aroma notes. 'N36' had strong sweet and woody aroma, respectively.

To have an insight into the reasons behind this observation, the different pineapple varieties were subjected to AEDA and GC-O.

Table 1 The mean scores and relative standard deviation of the seven aroma-attributes for the six pineapple varieties grown in Malaysia

\begin{tabular}{|c|c|c|c|c|c|c|c|}
\hline \multicolumn{3}{|l|}{ Fruit } & \multicolumn{5}{|l|}{ Mean values } \\
\hline & Sweet (RSD \%) & Floral (RSD \%) & Fruity (RSD \%) & Fresh (RSD \%) & Green (RSD \%) & Woody (RSD \%) & Apple-like (RSD \%) \\
\hline Moris & $8.50^{b}(2.8)$ & $5.67^{b}(4.0)$ & $6.83^{\mathrm{a}}(2.2)$ & $7.31^{\mathrm{a}}(4.5)$ & $3.85^{\mathrm{e}}(4.8)$ & $5.63^{d}(5.9)$ & $6.81^{b}(5.7)$ \\
\hline Maspine & $6.81^{e}(2.6)$ & $2.56^{f}(3.1)$ & $4.40^{f}(1.2)$ & $6.75^{b}(4.9)$ & $6.00^{b}(5.6)$ & $4.00^{f}(4.8)$ & $6.15^{c}(5.3)$ \\
\hline MD2 & $8.62^{\mathrm{a}}(2.9)$ & $6.88^{\mathrm{a}}(3.7)$ & $6.40^{\mathrm{b}}(1.1)$ & $6.05^{c}(4.1)$ & $2.57^{f}(3.4)$ & $5.15^{\mathrm{e}}(3.1)$ & $8.31^{\mathrm{a}}(2.6)$ \\
\hline N36 & $7.82^{d}(3.3)$ & $4.66^{c}(4.1)$ & $5.13^{\mathrm{e}}(4.3)$ & $4.75^{e}(4.6)$ & $5.26^{c}(4.7)$ & $6.05^{c}(3.0)$ & $4.15^{\mathrm{e}}(3.4)$ \\
\hline Josapine & $8.01^{e}(4.0)$ & $3.58^{\mathrm{d}}(3.0)$ & $5.05^{d}(3.7)$ & $5.35^{\mathrm{d}}(5.5)$ & $4.50^{d}(5.3)$ & $6.91^{b}(5.0)$ & $5.34^{d}(4.5)$ \\
\hline Sarawak & $6.45^{f}(2.5)$ & $3.05^{\mathrm{e}}(2.7)$ & $5.52^{c}(1.6)$ & $4.54^{f}(2.3)$ & $7.62^{\mathrm{a}}(4.4)$ & $7.46^{\mathrm{a}}(3.7)$ & $3.56^{f}(2.1)$ \\
\hline
\end{tabular}

Superscripts with different letters are significantly $(p<0.05)$ different 


\section{Characterization of aroma-active compounds by GC-O analysis}

A total of 59 volatile compounds were detected in the six different pineapple varieties grown in Malaysia (Table 2). The details are listed in Table 2. Pineapple 'Moris' had the highest number of compounds with a total of 31 compounds and this was followed by 'MD2' with 27 aroma-active compounds. The next were 'N36', 'Maspine', and 'Sarawak' which produced 24, 20 and 18 aromaactive compounds respectively. 'Josapine had the least number (16) of aroma-active compounds. Some of the compounds detected were methyl-2-methylbutanoate, dimethyl malonate, methyl-2-methyl acetoacetate, methyl-2-hydroxy-2-methylbutanoate, methyl hexanoate, ethyl isohexanoate, methyl-2-methylhexanoate, methyl3-(methylthiol)-propanoate, ethyl hexanoate, y-lactone, 2,5-dimethyl-4-hydroxy-3(2H)-furanone, methyl-3-hydroxyhexanoate, 2,5-dimethyl-4-methoxy-3(2H)furanone, methyl octanoate, methyl-(4E)-octenoate, 2,4-dihydroxy-2,5-dimethyl-3(2H)-furanone. Among the aforementioned compounds, 12 aroma-active compounds with flavour dilution $(F D) \geq 16$ were identified as key odorants through the application of the aroma extract dilution analysis (AEDA) (Table 2). For all the pineapple varieties, the highest FD factor was attributed to methyl-2-methylbutanoate (FD, 1024), methyl hexanoate (FD, 128) and 2,4-dihydroxy-2,5-dimethyl-3(2H)furanone (DMHF) (FD, 128), respectively.

Meanwhile, methyl-2-methylbutanoate which exhibited the highest FD factor had a bigger influence on the aroma profile of pineapple 'Moris'. It was however, not detected in the other varieties. On the other hand, methyl hexanoate and DMHF contributed significantly to the aroma profiles of the different pineapple varieties. This observation was similar to those of Zheng et al. [3]. For instance, the FD factors of methyl hexanoate in the different pineapple varieties were 64, 128, 64, 32 and 16 corresponding to 'Moris', 'MD2', 'N36,' 'Josapine' and 'Sarawak'. 2,4-Dihydroxy-2,5-dimethyl-3(2H)-furanone had greater influence on the aroma profiles of "Moris' 'Maspine' and 'MD2' with a corresponding FD factors of 16, 64 and 128, respectively. In addition, aroma-active compounds with relatively high FD factors such as $\delta$-octalactone, 2-methoxy-4-vinyl phenol, methyl octanoate and hexadecanoic acid had appreciable influence on the aroma profile of the pineapple varieties (Table 2).

\section{Quantitation of aroma-active compounds}

The detected aroma-active compounds and their mean concentrations were listed in Table 3. Most of the aroma-active compounds were branched esters. Recently, Steingrass et al. [12, 21] also reported that esters were the main volatile compounds in fresh pineapple, which is in agreement with our findings. In addition, several other groups of compounds such as ketones, alcohols, terpenes, lactones and acids were detected in the different pineapple varieties. Branched esters such as methyl-2-methyl butanoate, methyl2-methyl pentanoate, ethyl-2,3-dimethylbutanoate, methyl-2-methyl acetoacetate, methyl-2-hydroxy2-methylbutanoate, methyl-3-(methylthiol)-propanoate, methyl-3-hydroxy-4-methylpentanoate, methyl hexanoate, and methyl-3-hydroxyhexanoate were the most abundant compounds. Among these compounds, methyl-3-(methylthiol)-propanoate $(307 \pm 9.7 \mu \mathrm{g} / \mathrm{kg})$ methyl-2-methylbutanoate $(103 \pm 8.5 \mu \mathrm{g} / \mathrm{kg})$, methyl2-hydroxy-methylbutanoate $(86.0 \pm 6.5 \mu \mathrm{g} / \mathrm{kg})$, methyl3-hydroxy-4-methyl pentanoate $(65.0 \pm 5.6 \mu \mathrm{g} / \mathrm{kg})$, methyl hexanoate $(397 \pm 15 \mu \mathrm{g} / \mathrm{kg})$ and methyl-2-methyl acetoacetate $(156.1 \pm 12.0 \mu \mathrm{g} / \mathrm{kg})$ produced higher concentrations than other esters in the pineapple varieties (Table 3). However, research to determine the mechanism by which these esters are generated has been limited. The primary enzyme believed to be responsible for ester production is the alcohol acyltransferase (AAT), which was first isolated from 'Chandler' fruit [25].

Whilst methyl-branched esters such as methyl-2-methyl butanoate, methyl-2-methylpentanoate, etc. are assumed to be derived from branched-chain amino acid catabolism [25], Methyl-3-(methylthiol)-propanoate which exhibited high concentrations in 'Moris', 'MD2' and 'Sarawak' has been attributed to the Stickland reactions of methionine [26]. It is worthy of note that the ethyl derivatives of odd numbered carboxylic acids or branched carboxylic acids such as ethyl-2,3-dimethylbutanoate, ethyl isohexanoate and ethyl hexanoate were more specific and appeared in appreciable amount in pineapple 'Moris' only (Table 3). Furthermore, 'Moris' was also characterized by several acetates and acetoxy esters such as methyl-2-methyl acetoacetate, methyl butyl acetate, methyl-5-acetoxy octanoate and 3-octyl acetate. The acetates probably resulted from the condensation of acetyl-CoA with alcohols and hydroxyl-fatty acids [25]. Earlier on Steingass et al. [25] postulated that accumulation of acetyl-CoA under anaerobic condition can facilitate the production of both acetates and acetoxylated esters. To corroborate this position, alcohol acetyl transferase (AATs) enzymes' involvement in the genesis of acetates have been reported in different fruits such as; apples, bananas, pineapples and melon [16]. In addition, there was a marked dominance of the furanones (i.e. 2,5-dimethyl-4-hydroxy-3(2H)furanone; 2,4-dihydroxy-2,5-dimethyl-3(2H)-furanone) and lactones (i.e. $\mathrm{y}$-lactone, $\delta$-lactone, $\mathrm{y}$-octalactone, and $\delta$-octalactone) in 'Moris' as compared to the other pineapple varieties. 
Table 2 Detected aroma compounds with retention index and mean concentration ( $\mu \mathrm{g} / \mathrm{kg}$ fresh fruit) found in each pineapple varieties grown in Malaysia

\begin{tabular}{|c|c|c|c|c|c|c|c|c|c|}
\hline No & Compound $^{a}$ & Aroma-quality $^{\mathbf{b}}$ & Moris & Maspine & MD2 & N36 & Josapine & Sarawak & RI on TG-5 ms \\
\hline $\mathrm{C} 1$ & Methyl-2-methylbutanoate & Apple-like & $103 \pm 8.5$ & - & - & - & - & - & $771[770][31]$ \\
\hline C2 & 2-Hexanol & Winey & $2.1 \pm 0.0$ & - & - & - & - & $1.0 \pm 0.0$ & 780 [786] [32] \\
\hline C3 & 3-Methylbutanoic acid & Cheesy & - & - & $21.0 \pm 1.5$ & - & - & - & 792 \\
\hline C4 & Methyl butyl acetate & Banana & $8.0 \pm 1.0$ & - & - & - & - & - & 812 \\
\hline C5 & Methyl-2-methylpentanoate & Fruity & $7.3 \pm 1.2$ & - & - & - & - & $6.7 \pm 0.1$ & $823[\mathrm{nf}]$ \\
\hline C6 & Gamma-butyrolactone & Milky & - & - & $3.0 \pm 0.1$ & - & - & - & 837 \\
\hline C7 & Dimethyl malonate & Fruity & $48.2 \pm 3.5$ & - & $2.0 \pm 0.0$ & - & - & $2.0 \pm 0.0$ & $843[\mathrm{nf}]$ \\
\hline $\mathrm{C} 8$ & Ethyl-2,3-dimethylbutanoate & Fruity & $1.5 \pm 0.0$ & - & - & - & - & - & 856 [856] [32] \\
\hline C9 & Methyl-2-methyl acetoacetate & Fruity & $156.1 \pm 12.0$ & - & - & $13.0 \pm 1.5$ & - & - & $868[\mathrm{nf}]$ \\
\hline $\mathrm{C} 10$ & $\begin{array}{l}\text { Methyl-2/3-hydroxy-2/3-meth- } \\
\text { ylbutanoate }\end{array}$ & Fruity & $86.0 \pm 6.5$ & - & $7.0 \pm 0.1$ & - & - & - & 877 \\
\hline C11 & Methyl hexanoate & Fruity & $397 \pm 15.0$ & $\operatorname{tr}$ & $44.0 \pm 2.1$ & $19.0 \pm 0.1$ & $\operatorname{tr}$ & $32.0 \pm 1.0$ & 884 \\
\hline $\mathrm{C} 12$ & Ethyl isohexanoate & Pineapple & $13.0 \pm 1.0$ & - & - & - & - & - & 920 \\
\hline C13 & Methyl-2-methylhexanoate & Fruity & - & - & $8.0 \pm 0.1$ & - & - & - & 931 \\
\hline C14 & $\begin{array}{l}\text { Methyl-3-(methylthiol)-pro- } \\
\text { panoate }\end{array}$ & Sulphurous & $307 \pm 9.7$ & - & $28.7 \pm 1.0$ & - & - & $17.0 \pm 0.1$ & 936 \\
\hline C15 & Hexanoic acid & Fatty & - & - & $12.4 \pm 0.1$ & - & - & - & 974 [975] [32] \\
\hline C16 & (E)- $\beta$-Ocimene & Sweet/herbal & $4.0 \pm 0.0$ & - & $1.0 \pm 0.0$ & - & $2.0 \pm 0.0$ & $1.0 \pm 0.0$ & 976 \\
\hline C17 & $\begin{array}{l}\text { Methyl-3-hydroxy-4-methyl- } \\
\text { pentanoate }\end{array}$ & Fruity & $65.0 \pm 5.6$ & - & - & - & - & - & 983 \\
\hline C18 & Ethyl hexanoate & Fruity & $13.0 \pm 1.2$ & - & - & - & - & $1.0 \pm 0.0$ & 984 [1002] [32] \\
\hline C19 & Gamma-lactone & Creamy & $202.0 \pm 9.7$ & - & - & - & $11.0 \pm 0.1$ & $5.0 \pm 0.1$ & 986 [986] [32] \\
\hline $\mathrm{C} 20$ & Delta-lactone & ND & $221 \pm 11.0$ & - & - & $15.1 \pm 1.2$ & $9.0 \pm 0.1$ & $5.6 \pm 0.1$ & 1006 \\
\hline C21 & $\begin{array}{l}\text { 2,5-Dimethyl-4-hydroxy-3(2H)- } \\
\text { furanone }\end{array}$ & Strawberry & $55.0 \pm 3.4$ & $9.0 \pm 1.0$ & $1.5 \pm 0.0$ & $1.2 \pm 0.0$ & $54.2 \pm 2.0$ & $6.0 \pm 0.1$ & 1022 \\
\hline $\mathrm{C} 22$ & Methyl-3-hydroxyhexanoate & Fruity & - & - & $11.2 \pm 0.1$ & - & - & - & 1047 \\
\hline C23 & $\begin{array}{l}\text { 2,5-Dimethyl-4-methoxy- } \\
\text { 3(2H)-furanone }\end{array}$ & Roasty/sweet & - & - & $7.4 \pm 0.1$ & - & - & - & 1055 \\
\hline C24 & Methyl octanoate & Fruity & $101.0 \pm 8.0$ & - & $3.0 \pm 0.0$ & - & - & $4.0 \pm 0.1$ & 1083 \\
\hline C25 & Methyl (4E)-4-octenoate & Fruity & $30.0 \pm 3.0$ & - & - & - & - & - & 1091 \\
\hline C26 & 3-Octyl acetate* & Herbal/green & - & - & $2.0 \pm 0.0$ & - & - & - & 1118 [1119] [32] \\
\hline C27 & $\begin{array}{l}\text { 2,4-Dihydroxy-2,5-dimethyl- } \\
\text { 3(2H)-furanone }\end{array}$ & Fruity & $2.0 \pm 0.0$ & $3.2 \pm 0.1$ & $4.3 \pm 0.1$ & - & - & - & 1173 \\
\hline C28 & Octanoic acid & Rancid & $5.0 \pm 0.1$ & $2.0 \pm 0.0$ & $2.1 \pm 0.0$ & - & - & - & 1174 \\
\hline C29 & Gamma-octalactone & Coconut-like & $86.2 \pm 4.0$ & - & - & - & - & - & 1184 \\
\hline C30 & Delta-octalactone & Creamy & $11.0 \pm 1.5$ & - & $3.5 \pm 0.0$ & $3.0 \pm 0.1$ & $11.0 \pm 0.1$ & $7.0 \pm 0.1$ & 1205 \\
\hline C31 & Copaene & Woody & $40.1 \pm 3.9$ & - & $12.0 \pm 1.2$ & - & $3.0 \pm 0.1$ & - & 1221 \\
\hline C32 & Methyl decanoate & Floral & $4.0 \pm 0.1$ & - & - & - & - & - & 1282 \\
\hline C33 & 2-Methoxy-4-vinyl phenol & Smoky & - & $4.0 \pm 0.1$ & $2.0 \pm 0.0$ & $18.0 \pm 1.0$ & - & - & 1293 \\
\hline C34 & Decanoic acid & Sweaty & - & $2.0 \pm 0.0$ & $2.0 \pm 0.0$ & - & $2.0 \pm 0.0$ & - & 1372 \\
\hline C35 & Methyl-5-acetoxy octanoate & Wine-like & $5.0 \pm 0.1$ & - & - & - & - & - & 1385 \\
\hline C36 & gamma-Farnesene & ND & - & - & $2.7 \pm 0.0$ & - & - & - & 1453 \\
\hline C37 & Delta-undecalactone* & Coconut-like & - & - & $2.0 \pm 0.1$ & - & $4.1 \pm 0.1$ & - & 1483 [1488] [33] \\
\hline C38 & Germacrene & Woody & - & - & $1.0 \pm 0.0$ & - & - & - & 1515 [1502] [33] \\
\hline C39 & Globulol & Floral & - & - & $2.0 \pm 0.1$ & - & - & - & 1530 \\
\hline C40 & (-)-Spathulenol & Earthy & $19.0 \pm 1.0$ & - & $8.0 \pm 1.5$ & - & - & - & 1536 \\
\hline C41 & Dodecanoic acid & Sweaty/soapy & $7.0 \pm 0.1$ & - & - & $3.0 \pm 0.1$ & - & - & 1570 \\
\hline C42 & Gamma-dodecalactone & Fruity & - & - & - & $1.0 \pm 0.0$ & - & - & 1582 [1587] [32] \\
\hline C43 & (Z)-7-Tetradecenal & ND & - & $1139 \pm 34.0$ & - & - & - & - & 1609 \\
\hline C44 & Pentadecanal* & Fresh/waxy & $18.1 \pm 1.0$ & - & - & $4.0 \pm 0.1$ & - & - & $1701[1712][36]$ \\
\hline
\end{tabular}


Table 2 (continued)

\begin{tabular}{|c|c|c|c|c|c|c|c|c|c|}
\hline No & Compound $^{a}$ & Aroma-quality $^{\mathbf{b}}$ & Moris & Maspine & MD2 & N36 & Josapine & Sarawak & RI on TG-5 ms \\
\hline C45 & $\begin{array}{l}\text { 3,5-Dimethoxy-4-hydroxycin- } \\
\text { namaldehyde }\end{array}$ & Cocoa-like & - & $3.0 \pm 0.1$ & - & - & - & - & 1788 \\
\hline C46 & Pentadecanoic acid & Waxy & - & $3.0 \pm 0.1$ & - & $2.0 \pm 0.0$ & - & $1.0 \pm 0.0$ & 1869 \\
\hline C47 & Methylhexadecanoate* & Waxy & - & $2.6 \pm 0.0$ & - & - & - & $1.0 \pm 0.0$ & $1878[1878][32]$ \\
\hline C48 & Methyl-(2E)-2-hexadecenoate & ND & - & $8.0 \pm 0.1$ & - & - & - & - & 1886 \\
\hline C49 & Ethyl hexadecanoate & Waxy & - & $4.0 \pm 0.1$ & - & - & $1.0 \pm 0.0$ & - & 1928 \\
\hline C50 & Hexadecanoic acid* & Waxy & - & $51.7 \pm 3.2$ & $255.0 \pm 9.0$ & $5.0 \pm 0.1$ & $393.0 \pm 11.2$ & $2.0 \pm 0.0$ & 1968 [1970] [32] \\
\hline C51 & 9-Hexadecenoic acid & Waxy & - & $2.0 \pm 0.0$ & - & - & - & - & 1976 \\
\hline C52 & Octadecanal & Fatty/greasy & - & - & - & $21.0 \pm 1.5$ & - & - & 1999 [2002] [32] \\
\hline C53 & Eicosane & ND & $105.1 \pm 9.0$ & $2.0 \pm 0.0$ & - & $14.0 \pm 2.0$ & $2.0 \pm 0.0$ & $16.0 \pm 1.0$ & 2009 \\
\hline C54 & Heptadecanoic acid* & Waxy & - & $4.0 \pm 0.1$ & - & - & $3.0 \pm 0.1$ & - & 2067 [2067] [32] \\
\hline C55 & Octadecanoic acid* & Pungent & - & $149.0 \pm 9.0$ & $1.0 \pm 0.0$ & $69.0 \pm 5.1$ & - & $89.0 \pm 7.0$ & 2167 [2167] [32] \\
\hline C56 & Ethyl octadecanoate* & Waxy & - & $46.0 \pm 3.0$ & - & - & $2.0 \pm 0.0$ & - & 2177 [2174] [33] \\
\hline C57 & $\begin{array}{l}\text { (Z,Z)-9,12-Octadecadienoic } \\
\text { acid* }^{*}\end{array}$ & Waxy & - & $37.0 \pm 2.1$ & - & - & $37.0 \pm 4.0$ & $16.0 \pm 1.5$ & 2183 [2183] [36] \\
\hline C58 & Ethyl oleate ${ }^{*}$ & Fatty & - & $89.0 \pm 6.5$ & - & - & $57.0 \pm 2.0$ & - & 2185 [2180] [32] \\
\hline C59 & Geranyl geraniol & Floral & $11.0 \pm 0.1$ & - & - & - & - & - & 2192 \\
\hline
\end{tabular}

- Odorant not detected

ND not detectable

$\operatorname{tr}$ Trace $(<1.0 \mu \mathrm{g} / \mathrm{kg}),\left[\mathrm{RI}_{\text {lit }}{ }^{35}\right.$; Scheidig et al. [31], [RI lit ${ }^{36} ;$ NIST [32], [RI ${ }_{\text {lit }}{ }^{37 ;}$ El-Sayad [33]

a Compounds were identified by comparing their retention indices on the TG-5 ms column, their mass spectra, and odour nuances with the respective data of the reference odorants

b Aroma-quality perceived by panellists during olfactometry

* Compounds tentatively identified with the MS database and retention index

Surprisingly, $\delta$-undecalactone was mainly detected in 'MD2' and 'Josapine'. Lactones which exhibited creamy and coconut-like aroma notes in the pineapple varieties have been identified as most potent odorants in pineapples [27]. The formation of lactones in fruits has been documented. There are two proposed pathways for the formation of lactones [28]. The first pathway is from unsaturated fatty acids to lactones via hydroperoxy fatty acids and monohydroxy fatty acids under the actions of lipoxygenase (LOX) and peroxygenase (PGX). The second pathway is from unsaturated fatty acids to lactones via epoxy fatty acids and dihydroxy fatty acids under the actions of PGX and epoxide hydrolase. 4-Hydroxy-2,5-dimethyl-3(2H)-furanone and its methyl ether 2,5-dimethyl-4-methoxy-3(2H)-furanone are important odorants of many fruits [29]. Whereas, 4-hydroxy-2,5-dimethyl$3(2 \mathrm{H})$-furanone and its derivatives are synthesized by a series of enzymatic steps in fruits, they are also products of Maillard reaction [30].

\section{Relationship between pineapple varieties and odour-active compounds}

In order to differentiate between the six different pineapples in terms of the aroma-active compounds associated with each variety, principal component analysis
(PCA) was used. PCA provides a visual relationship between the pineapple varieties and their aroma-active compounds. This method makes the interpretation of the multivariate analysis easy. A first PCA was performed on the concentration of the 59 volatile compounds (Table 2) analysed in the pineapple varieties. Based on the samples grouping from PCA, a partial least square discriminant analysis (PLS-DA) was established (Fig. 1a). The scatter plot of scores of the first two components (in PLS-DA which explained 95\% of the total variance in the data) showed the differences among the six pineapple varieties. The corresponding PLS weight plot (Fig. 1b) revealed the inter-relationship between the aroma compounds and the pineapple varieties.

Malaysian pineapples were separated according to their varieties (Fig. 1a). Low negative component 1 and high positive component 2 corresponded to pineapple 'MD2'. The pineapple variety 'Maspine' was situated within low negative components 1 and 2, respectively. While pineapple 'Moris' was within the area of high positive component 1 and low negative component 2, other varieties such as 'Sarawak', Josapine and N36, were all situated at the region of low negative component 1 and low positive component 2. 
Table 3 Detected aroma compounds with their flavour dilution (FD) factors in each pineapple varieties (Moris, Maspine, MD2, N36, Josapine and Sarawak) grown in Malaysia

\begin{tabular}{|c|c|c|c|c|c|c|c|c|c|}
\hline No & Compound $^{a}$ & Aroma-quality $^{\mathbf{b}}$ & Moris & Maspine & MD2 & N36 & Josapine & Sarawak & RI on TG-5 ms \\
\hline 1 & Methyl-2-methylbutanoate & Fruity & 1024 & - & - & - & - & - & 771 \\
\hline 2 & 2-Hexanol & Winey & 2 & - & - & - & - & 2 & 780 \\
\hline 3 & 3-Methylbutanoic acid & Cheesy & - & - & 2 & - & - & - & 792 \\
\hline 4 & Methyl butyl acetate & Banana & 2 & - & - & - & - & - & 812 \\
\hline 5 & Methyl-2-methylpentanoate & Fruity & 4 & - & - & - & - & 2 & 823 \\
\hline 6 & Gamma-butyrolactone & Weak, milky & - & - & 2 & - & - & - & 837 \\
\hline 7 & Dimethyl malonate & Fruity & 8 & - & 2 & - & - & 2 & 843 \\
\hline 8 & Ethyl-2,3-dimethylbutanoate & Fruity & 8 & - & - & - & - & - & 856 \\
\hline 9 & Methyl-2-methyl acetoacetate & Fruity & 8 & - & - & 8 & - & - & 868 \\
\hline 10 & Methyl-2/3-hydroxy-2/3-methylbutanoate & Fruity & 8 & - & 4 & - & - & - & 877 \\
\hline 11 & Methyl hexanoate & Fruity & 64 & - & 128 & 64 & 32 & 16 & 884 \\
\hline 12 & Ethyl isohexanoate & Pineapple & 8 & - & - & - & - & - & 920 \\
\hline 13 & Methyl-2-methylhexanoate & Sulfurous & 8 & - & 4 & - & - & 2 & 931 \\
\hline 15 & Hexanoic acid & Fatty & - & - & 2 & - & - & - & 974 \\
\hline 16 & (E)- $\beta$-Ocimene & Sweet, herbal & 2 & - & 2 & - & 2 & 2 & 976 \\
\hline 17 & Methyl-3-hydroxy-4-methylpentanoate & Fruity & 8 & - & - & - & - & - & 983 \\
\hline 18 & Ethyl hexanoate & Fruity & 16 & - & - & - & - & 16 & 984 \\
\hline 19 & Gamma-lactone & Creamy & 16 & - & - & - & 16 & 8 & 986 \\
\hline 20 & Delta-lactone & ND & - & - & - & - & - & - & 1006 \\
\hline 21 & 2,5-Dimethyl-4-hydroxy-3(2H)-furanone & Strawberry & 16 & 16 & - & - & 32 & 16 & 1022 \\
\hline 22 & Methyl-3-hydroxyhexanoate & Fruity & - & - & 8 & - & - & - & 1047 \\
\hline 23 & 2,5-Dimethyl-4-methoxy-3(2H)-furanone & Caramel, sweet & - & - & 32 & - & - & - & 1055 \\
\hline 24 & Methyl octanoate & Fruity & 32 & - & 16 & - & - & 16 & 1083 \\
\hline 25 & Methyl (4E)-4-octenoate & Fruity & 8 & - & - & - & - & - & 1091 \\
\hline 26 & 3-Octyl acetate & Herbal/green & - & - & 2 & - & - & - & 1118 \\
\hline 27 & 2,4-Dihydroxy-2,5-dimethyl-3(2H)-furanone & Fruity & 16 & 64 & 128 & - & - & - & 1173 \\
\hline 28 & Octanoic acid & Rancid & 2 & 2 & 2 & - & - & - & 1174 \\
\hline 29 & Gamma-octalactone & Coconut & 4 & - & - & - & - & - & 1184 \\
\hline 30 & Delta-octalactone & Creamy & 16 & - & 32 & 16 & 16 & 16 & 1205 \\
\hline 31 & Copaene & Woody & 8 & - & 8 & - & 2 & - & 1221 \\
\hline 32 & Methyl decanoate & Floral & 2 & - & - & - & - & - & 1282 \\
\hline 33 & 2-Methoxy-4-vinyl phenol & Smoky & - & 16 & 4 & 32 & - & - & 1293 \\
\hline 34 & Decanoic acid & Sweaty & - & 2 & 2 & - & 2 & & 1372 \\
\hline 35 & Methyl-5-acetoxy octanoate & Winey & 8 & - & - & - & - & - & 1385 \\
\hline 36 & gamma-Farnesene & ND & - & - & - & - & - & - & 1453 \\
\hline 37 & Delta-undecalactone & Coconut & - & - & 32 & - & 16 & - & 1483 \\
\hline 38 & Germacrene & Woody & - & - & 2 & - & - & - & 1515 \\
\hline 39 & Globulol & Floral & - & - & 4 & - & - & - & 1530 \\
\hline 40 & (-)-Spathulenol & Earthy & 8 & - & 8 & - & - & - & 1536 \\
\hline 41 & Dodecanoic acid & Soapy/sweaty & 2 & - & - & 4 & - & - & 1570 \\
\hline 42 & $y$-Dodecalactone & Fruity & - & - & - & 2 & - & - & 1582 \\
\hline 43 & (Z)-7-Tetradecenal & $N D$ & - & - & - & - & - & $=$ & 1609 \\
\hline 44 & Pentadecanal & Waxy/fresh & 4 & - & - & 4 & - & - & 1701 \\
\hline 45 & 3,5-Dimethoxy-4-hydroxycinnamaldehyde & Cocoa-like & - & 2 & - & - & - & - & 1788 \\
\hline 46 & Pentadecanoic acid & Waxy & - & 2 & - & 2 & - & 2 & 1869 \\
\hline 47 & Methylhexadecanoate & Waxy & - & 4 & - & 4 & - & 4 & 1878 \\
\hline 48 & Methyl-(2E)-2-hexadecenoate & ND & - & - & - & - & - & - & 1886 \\
\hline 49 & Ethyl hexadecanoate & Waxy & - & 2 & - & - & 2 & - & 1928 \\
\hline
\end{tabular}


Table 3 (continued)

\begin{tabular}{|c|c|c|c|c|c|c|c|c|c|}
\hline No & Compound $^{a}$ & Aroma-quality ${ }^{\mathbf{b}}$ & Moris & Maspine & MD2 & N36 & Josapine & Sarawak & RI on TG-5 ms \\
\hline 50 & Hexadecanoic acid & Waxy/sweaty & - & 4 & 64 & 4 & 32 & 2 & 1968 \\
\hline 51 & 9-Hexadecenoic acid & Waxy & - & 2 & - & - & - & - & 1976 \\
\hline 52 & Octadecanal & Greasy & - & - & - & 4 & - & - & 1999 \\
\hline 53 & Eicosane & ND & - & - & - & - & - & - & 2009 \\
\hline 54 & Heptadecanoic acid & Waxy & - & 2 & - & - & 2 & - & 2067 \\
\hline 55 & Octadecanoic acid & Pungent/sweaty & - & 8 & 2 & 4 & - & 8 & 2167 \\
\hline 56 & Ethyl octadecanoate & Waxy & - & 8 & - & - & 2 & - & 2177 \\
\hline 57 & $(Z, Z)-9,12-$ Octadecadienoic acid & Waxy/sweaty & - & 8 & - & - & 8 & 4 & 2183 \\
\hline 58 & Ethyl oleate & Fatty & - & 8 & - & - & 8 & - & 2185 \\
\hline 59 & Geranyl geraniol & Floral & 8 & - & - & - & - & - & 2192 \\
\hline
\end{tabular}

$N D$ not detectable, FD Flavour dilution factor determined in extract containing the juice volatiles

- odorant not detected

a Compounds were identified by comparing their retention indices on the TG-5 ms column, their mass spectra, and odour nuances with the respective data of the reference odorants

b Aroma-quality perceived by panellists during olfactometry

In addition, the inter-relationship between the aroma-active compounds and the pineapple varieties were carried out by the partial least square (PLS)weight plot (Fig. 1b). The results revealed that 'Moris' covaried with 31 aroma-active compounds, majority of which were the fruity esters with $F D \geq 8$ such as methyl-2-methylbutanoate (C1), methyl butyl acetate (C4), ethyl-2,3-dimethylbutanoate (C8), ethyl iso hexanoate (C12), methyl-3-hydroxy-4-methylpentanoate (C17), 2,5-dimethyl-4-hydroxy-3(2H) furanone (C21), methyl octanoate (C25), methyl-5-acetoxy octanoate (C35) and geranyl geraniol (C59) (Table 3) and (Fig. 2). Similarly, 'Moris' also covaried with other compounds such as y-octalactone (C29), $\delta$-octalactone (C30), and (-)-spathulenol (C40). On the other hand, 'Maspine' was correlated with 2-methoxy-4-vinyl-phenol (C33), (Z)-7-tetradecenal (C43), 3,5-dimethoxy-4-hydroxycinnamaldehyde (C45), pentadecanoic acid (C46), methyl hexadecanoate (C47) and octadecanoic acid (C55) (Fig. 2). In the case of 'Sarawak', 'Josapine' and 'N36', they covaried with ethyl hexanoate $(\mathrm{C} 18))$, y-lactone (C42)), methyl octanoate (C24), $\delta$-octalactone (C20), and 2-methoxy-4-viny phenol (C33). However, 'MD2' covered with methyl-3(methylthiol)-propanoate (C14), methyl3-hydroxyhexanoate (C22), 2,4-dihydroxy-2,5-dimethyl-3 $(2 \mathrm{H})$-furanone (C27), $\delta$-undecalactone (C37), (Z)-7-tetradecenal (C43), 3,5-dimetoxy-4-hydroxycinnamaldehyde (C45), methyl hexadecanoate (C47) and decanoic acid (C34).

In order to validate the results obtained by PCA analysis, a hierarchical cluster analysis (HCA) was carried out using Ward's method of agglomeration and Euclidean distances to evaluate similarity between varieties. The test was performed on the complete dataset, thus obtaining the dendrogram in Fig. 3. Three main groups of pineapple varieties were identified by HCA. The first group comprised pineapple 'Moris' and 'MD2' Fig. 3. This group was characterized by high numbers of aroma-compounds most especially the fruity esters. They contained some of the highly intense aroma-active compounds (FD $\geq 64$ ) such as methyl-2-methyl butanoate, methyl hexanoate, methyl-3-(methylthiol)-propanoate and 2,4-dihydroxy2,5-dimethyl-3 (2H)-furanone. The second group contained pineapple 'Maspine'. This group contained the least quantity of fruity esters. The third group included 'Sarawak,' 'Josapine' and 'N36. This group contained more of the fatty acid methyl esters.

\section{Conclusion}

Sensory evaluation, GC-O and GC-MS analysis were employed to elucidate the characteristic aroma of six pineapples varieties grown in Malaysia. Application of qualitative descriptive sensory analysis on the six pineapple varieties revealed seven quality terms such as sweet, floral, fruity, fresh, green, woody and apple-like. In addition, 97 aroma-active compounds were identified by GC-O and AEDA in the pineapple varieties. Of this, pineapple 'Moris' had the highest numbers of aroma-active compounds with a total of 31 compounds and this was followed by 'MD2' with 27 compounds. The next were the 'N36', 'Maspine', and 'Sarawak' which produced 24, 20 and 18 aroma-active compounds, respectively. 'Josapine' had the least number of aroma-active compounds (16). 


\section{a}

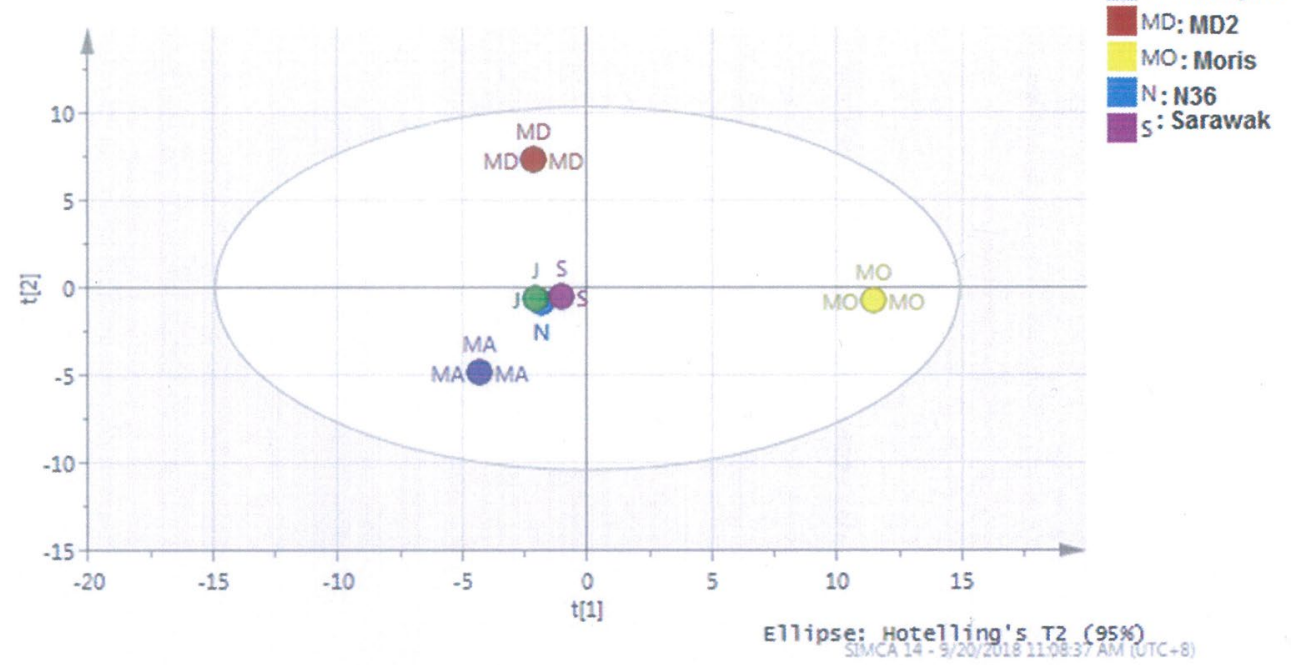

b

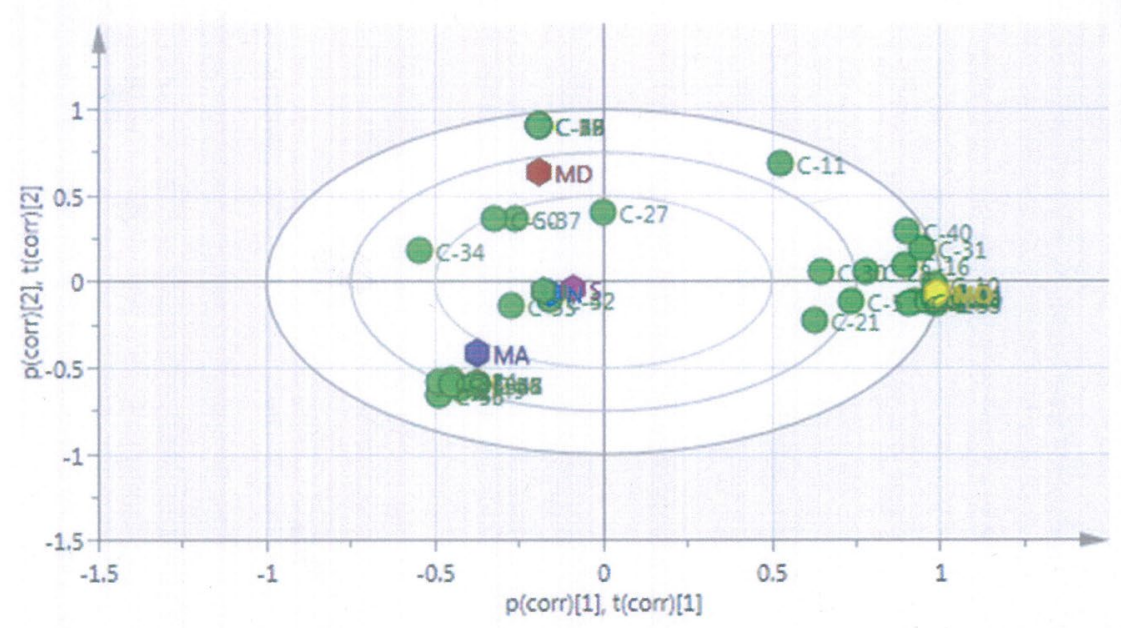

STMCA 14 - 9/20/2018 1109:20 AM (UTC+8)

Fig. 1 Score scatter PLS-DA and PLS weight plots $(\mathbf{a}, \mathbf{b})$ of the pineapple varieties grown in Malaysia, The PLS-DA plot shows similarities and differences in pineapple varieties while PLS-weight plot reveals the inter-relatedness between the fruits and 97 aroma-active compounds (P1-P97) shown in Table 2

In order to address the inter-relationship between the sensory attributes and the aroma compounds, the PLSR analysis was employed. Results of the analysis showed that 'Moris' and 'MD2' covaried majorly with the fruity esters with higher FD factors. 'Sarawak', 'Josapine' and 'N36' were correlated with fewer fruity esters; they covaried majorly with the lactones. However, the variety 'Maspine' was correlated with 2-methoxy-4-vinyl-phenol (C33), (Z)-7-tetradecenal (C43), 3,5-dimethoxy-4-hydroxycinnamaldehyde (C45), pentadecanoic acid (C46), methyl hexadecanoate (C47) and octadecanoic acid (C55), respectively. In addition, 

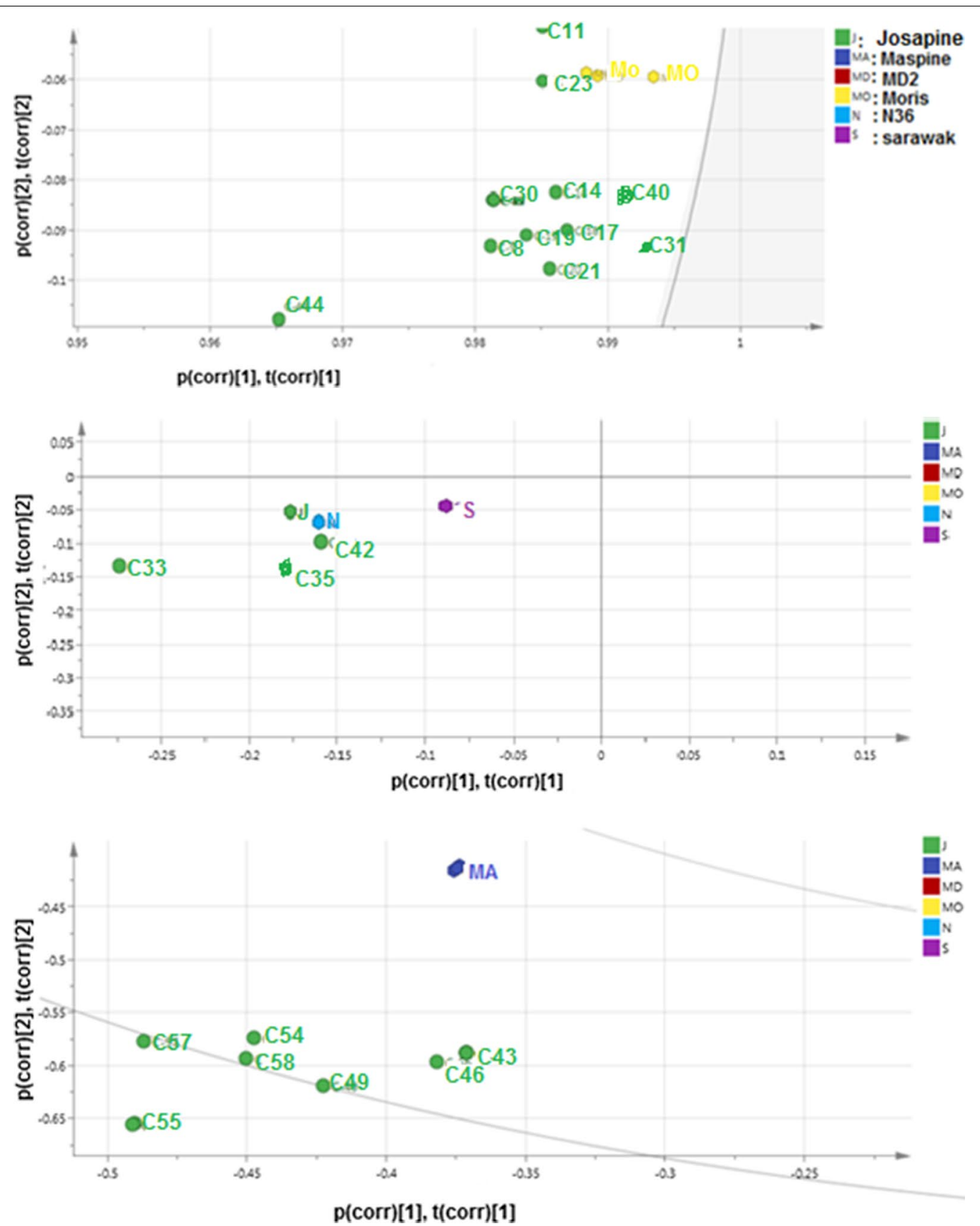

Fig. 2 Visualization of PLS weight plot of Fig. 1b. 1, 2 and 3 are aroma compounds correlating with Moris, (yellow), Sarawak, Josapine, N36 and Maspine respectively

hierarchical cluster analysis was used to establish similarities among the pineapples and the results revealed three main groups of pineapples.

\section{Experimental}

Pineapple fruits

Fresh, fully-ripe pineapples of six different varieties ('Moris', 'Maspine', 'MD2', 'N36', 'Josapine', and 'Sarawak') grown in Johor, Malaysia were obtained from an established farmer. Three fruits of each variety 


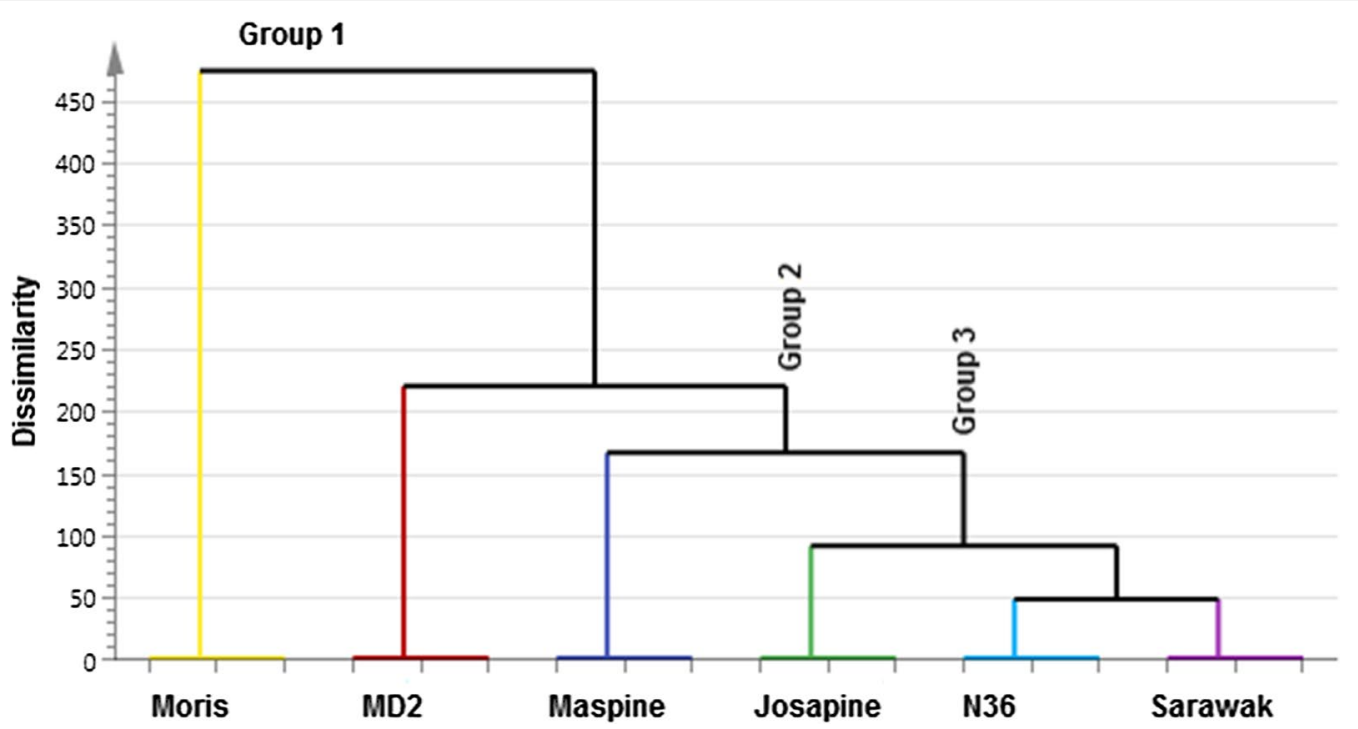

Fig. 3 Dendrogram of hierarchical cluster analysis of six pineapple varieties grown in Malaysia

were stored at $8 \pm 1{ }^{\circ} \mathrm{C}$ and $80-90 \%$ relative humidity until analysed. Fruits were selected with similar characteristics of ripening (i.e. pale-yellow skin colour; flat eyes; and degree of Brix), hand-peeled, cored, sliced and cut into small pieces before blending with a Panasonic Food Processor (model PSN-MKF300, Panasonic, Malaysia). One fruit weighed $927-1201 \mathrm{~g}$ apart from the crown. The $\mathrm{pH}$ and Brix values were 3.49, 3.50, 3.52, 3.54, 3.60, $10.33^{\circ}$ Brix, $11.45^{\circ}$ Brix, 12.48 ${ }^{\circ}$ Brix, $13.25^{\circ}$ Brix, $14.01^{\circ}$ Brix, and $16.50^{\circ}$ Brix for Sarawak, Maspine, N36, Josapine, Moris and MD2, respectively. At least three separate measurements were carried out for each analysis.

\section{Chemicals}

Pure reference standards of methyl-2-methylbutanoate (98.0\%), 2-hexanol (97.0\%), 3-methylbutanoic acid (97.5\%), methyl butyl acetate (98.0\%), methyl-2-methylpentanoate (99.5\%), gamma-butyrolactone (98.0\%), dimethyl malonate (97.0\%), ethyl-2,3-dimethylbutanoate (99.5\%), methyl-2-methyl acetoacetate (99.5\%), methyl2-hydroxy-2-methylbutanoate (98.0\%), methyl hexanoate (99.5\%), methyl-3-(methylthiol)-propanoate (99.5\%), hexanoic acid (97.0\%), trans- $\beta$-ocimene (98.0\%), methyl2-methylhexanoate (99.5\%), ethyl hexanoate (98.0\%), $\delta$-lactone $(98.0 \%), 2,5$-dimethyl-4-hydroxy-3(2H)-furanone (99.5\%), methyl-3-hydroxyhexanoate (99.5\%), 2,5-dimethyl-4-methoxy-3(2H)-furanone (98.0\%), methyl octanoate (99.5\%), octanoic acid (97.0\%), y-octalactone (98.5\%), $\delta$-octalactone (98.0\%), copaene (97.0\%), methyl decanoate (99.5\%), 2-methyl-4-vinyl phenol (99.5\%), decanoic acid (97.0\%), y-farnesene (98.0\%), germacrene
(98.0\%), globulol (98.0\%), spathulenol (98.0 5), (Z)7-tetradecenal (97.0\%), and octadecanal (99.5\%) were purchased from Aldrich, Steinheim, Germany. Gammalactone (98.0\%) and methyl dodecane (99.5\%) were obtained from Parchem, New Rochelle, NY and Achemica Corp. Aigle, Switzerland, respectively. The $n$-alkane standard $\left(\mathrm{C}_{7}-\mathrm{C}_{30}\right)$ was obtained from Sigma-Aldrich Chemicals Co. (St. Louis, MO). Other chemicals were of analytical grade.

\section{Isolation of pineapple volatile compounds}

The isolation of the pineapple volatile compounds was performed by extracting $300 \mathrm{~mL}$ of juice with dichloromethane $(300 \mathrm{~mL})$, followed by distillation in vacuum [34]. A similar workup procedure reported earlier [35] was carried out on juice to produce $400 \mu \mathrm{L}$ extract.

\section{GC-MS and GC-FID analyses}

The extracts were injected into a QP-5050A (Shimadzu, Kyoto, Japan) gas chromatograph equipped with a GC17A Ver.3, and a flame ionization detector (FID). Two microliters of the extract was vaporized in the injector port maintained at $220^{\circ} \mathrm{C}$ in split less mode $(1 \mathrm{~min})$. The oven temperature was varied from $50{ }^{\circ} \mathrm{C}$ to $250{ }^{\circ} \mathrm{C}$ at $15^{\circ} \mathrm{C} / \mathrm{min}$, and holding times of 3 and $10 \mathrm{~min}$ respectively [36]. A $30-300 \mathrm{~m} / \mathrm{z}$ mass range was recorded in full-scan mode. The quadrupole ion source and transfer line temperatures were maintained at 150 and $250{ }^{\circ} \mathrm{C}$. respectively and the ionisation energy was set at $70 \mathrm{eV}$. The column $(30 \mathrm{~m} \times 0.25 \mathrm{~mm}$ i.d., and $0.25 \mu \mathrm{m}$ film thickness; $5 \%$ diphenyl/95\% dimethylpolysiloxane phase; Thermo Scientific, Milan Italy) was a TG-5 ms [36]. The 
carrier gas was helium at $1.5 \mathrm{~mL} / \mathrm{min}$ (column-head pressure of $13 \mathrm{psi})$.

\section{GC-O analysis}

A Trace Ultra 1300 gas chromatograph (Thermos Scientific, Waltham, MA, USA) fitted with a TG- $5 \mathrm{~ms}$ column $(30 \mathrm{~m} \times 0.25 \mathrm{~mm}$ i.d., film thickness, $0.25 \mu \mathrm{m}$, Thermo Scientific, Milan Italy) and an ODP 3 olfactory Detector Port (Gerstel, Mulheim, Germany), with additional supply of humidified purge air, was operated as earlier reported by Lasekan [35]. The split ratio between the sniffing port and the FID detector was 1:1. Two replicate samples were sniffed by three trained panellists who presented normalized responses, with strong agreement with one another.

\section{Identification and quantification}

Kovats method which employs a mixture of normal paraffin $\mathrm{C}_{7}-\mathrm{C}_{30}$ as external references was used to calculate the linear retention indices [36]. The identification of compounds was as described by Lasekan and $\mathrm{Ng}$ [34]. When it was not possible to find appropriate reference standard, a tentative identification was obtained by matching retention index with mass spectral libraries data (WILEY 275, NBS75K). Semi-quantitative data were obtained by relating the peak area of each compound to that of the corresponding standard and were expressed as $\mu \mathrm{g} / \mathrm{kg}$. For compounds tentatively identified, their semi-quantitative data were obtained by relating their peak area to that of octadecane and were expressed as $\mu \mathrm{g} / \mathrm{kg}$ octadecane.

\section{Aroma extracts dilution analysis (AEDA)}

The flavor dilution (FD) factors of the aroma-active compounds were evaluated by GC-O using the AEDA approach earlier reported by Lasekan [35]. Each of the obtained dilution was injected into the GC-O. The highest dilution in which an aroma compound was observed is referred to as the flavor dilution (FD) factor of that compound [37].

\section{Sensory analysis}

Sensory analysis was carried out by ten trained panelists (6 females and 4 males) in a sensory laboratory according to the International Standard ISO 8589: [29]. All panelists who have passed screening test as described earlier [34] were recruited from the University Putra Malaysia. Prior to the test, the panelist were taken through $1 \mathrm{~h}$ training session with selected aroma compounds such as: ethyl hexanoate (fruity), 2,5-dimethyl-4-hydroxy$3(2 \mathrm{H})$-furanone (Strawberry), $\beta$-damascenone (floral), ethyl isohexanote (pineapple-like), etc. Descriptors used by panelists were determined after three preliminary sensory experiments. Finally, the panelists were asked to evaluate ortho-nasally fresh pineapple juice placed inside glass containers $(7 \mathrm{~cm} \times 3.5 \mathrm{~cm})$. Seven aroma attributes (sweet, floral, fruity, fresh, green, woody and apple-like) were obtained. Panelists were asked to score each attribute on a 10-point interval scale with $9=$ strong intensity, and $0=$ weak with no perception. To aid the sensory analysis, the following reference compounds: ethyl hexanote (fruity), $\beta$-damascenone (floral), methyl-3(methylthiol)propanoate (apple-like), hexanal (green), germacrene (woody), $p$-anisaldehyde (sweet) and (E,Z)-3,5-undecatriene (fresh, pineapple-like) were dissolved in water at a concentration of $100 \times$ above their respective threshold values. The fresh pineapple varieties were evaluated in triplicate and the results obtained were averaged.

\section{Statistical analysis}

Analysis of variance (ANOVA) and Duncan's multiple comparison tests were carried out to establish if statistical differences existed among individual pineapple variety for each sensory attribute at $(\mathrm{p}<0.05)$. Partial least square discriminate analysis (PLS-DA) and PLS-regression coefficient were employed as an exploratory tool to describe and summarise the data by grouping variables that are correlated. The mean concentrations of the 59 aroma-active compounds and the six different pineapple varieties (Table 3 ) were the data set. The multivariate statistical analyses were performed using the SIMCA-P software (V. 10.0, Umetricus, Umea, Sweden). Principal Components Analysis (PCA) and Hierarchical Cluster Analysis (HCA) using the Software package SPSS Statistics 17.0 (SPSS Inc., Chicago, IL) were also employed.

\section{Abbreviations \\ ANOVA: analysis of variance; PCA: principal component analysis; HCA: hierar- chical cluster analysis; FD: flavour dilution.}

\section{Authors' contributions}

$\mathrm{OL}$ conceptualized this study and critically review the content of the manuscript. FKH carried out the experiments, data analysis and interpretations. OL has made intellectual contributions. All authors read and approved the final manuscript.

\section{Acknowledgements \\ The authors are grateful for the extensive financial support received from the} University Putra Malaysia research scheme Grant (9478500).

\section{Competing interests}

The authors declare that they have no competing interests.

\section{Publisher's Note}

Springer Nature remains neutral with regard to jurisdictional claims in published maps and institutional affiliations.

Received: 17 October 2018 Accepted: 27 November 2018

Published online: 19 December 2018 


\section{References}

1. Jaji K, Man N, Nawi NM (2018) Factors affecting pineapple market supply in Johor, Malaysia. Int Food Res J 25:366-375

2. Food and Agriculture Organization (FAO). Pineapple fresh production http://faostat3.fao.org/home/index.html. Accessed 20 Mar 2018

3. Zheng LY, Sun GM, Liu YG, Lv LL, Yang WX, Zhao WF, Wei CB (2012) Aroma volatile compounds from two fresh pineapple varieties in China. Int J Mol Sci 13:7383-7392

4. Zemlicka L, Fodran P, Kolek E, Pronayova N (2013) Analysis of natural aroma and flavour of MD2 pineapple variety (A. comosus L. Merr). Acta Chim Slovaca 6:123-128

5. Steingass CB, Langen J, Carle R, Schmarr HG (2015) Authentication of pineapple (A. comosus L. Merr.) fruit maturity stages by quantitative analysis of gamma- and delta-lactones using headspace solid-phase micro extraction and chirospecific gas chromatography- selected ion monitoring mass spectrometry. Food Chem 168:496-503

6. Schwab W, Schreier P (2002) Enzymic formation of flavour volatiles from lipid. In: Kuo TM, Gardner HW (eds) Lipid biotechnology. Marcel Dekker, New York, pp 293-318

7. Baldwin IT, Kessler A, Halitschko R (2002) Volatile signalling in plant-herbivore interactions: what is real. J Curr Opin Plant Biol 5:351-354

8. Newman JD, Chappell J (1999) Isoprenoid biosynthesis in plants: carbon partitioning within the cytoplasmic pathway. Crit Rev Biochem Mol Biol 34:95-106

9. Rosati C, Diretto G, Giuliano G (2009) Biosynthesis and engineering of carotenoids and apocarotenoids in plants: state of the art and future prospects. Biotech Genet Eng Rev 26:151-174

10. Teai T, Claude-Lafontaine A, Schippa C, Cozzdino F (2001) Volatile compounds in fresh pulp of pineapple (A. comosus L. Merr) from French Polynesia. J Essent Oil 13:314-318

11. Liu SH, Wei CB, Sun GM, Zang XP (2008) Analysis of aroma components in 3 pineapple cultivars. Food Sci 29:614-617

12. Zhang XM, Du LQ, Sun GM, Liu SH, Wei CB, Liu ZH, Xie JH (2009) Analysis of aromatic components in pineapple varieties. Food Sci 30:275-279

13. Wei CB, Liu SH, Liu YG, Zhang XP, Lu LL, Sun GM (2011) Changes and distribution of aroma volatile compounds from pineapple fruit during postharvest storage. Acta Hortic 902:431-436

14. Robinson AL, Boss PK, Heymann H, Solomon PS, Trengrove RD (2011) Development of a sensitive non-targeted method for characterizing the wine volatile profile using head space solid-phase microextraction comprehensive two-dimensional gas chromatography time-of-flight mass spectrometry. J Chromotogr A 1218:504-517

15. Saurina J (2010) Characterization of wines using compositional profiles and chemometrics. Trends Anal Chem 29:234-245

16. Steingass CB, Jutzi M, Muller J, Carle R, Schmarr HG (2015) Ripeningdependent metabolic changes in the volatiles of pineapple (A. comosus L. Merr.) fruit: II. Multivariate statistical profiling of pineapple aroma compounds based on comprehensive two- dimensional gas chromatography mass spectrometry. Anal Bio Chem 407:2609-2624

17. Picariello G, Mamone G, Addeo F, Ferranti P (2012) Novel mass spectrometry-based applications of the 'Omic' sciences in food technology and biotechnology. Food Technol Biotechnol 50:286-305

18. Wibowo S, Grauwet T, Kebede BT, Hendrickx M, Loey AV (2015) Study of chemical in pasteurised orange juice during shelf-life: a finger printing-kinetics evaluation of the volatile changes fraction. Food Res Int 75:295-304

19. Dall'Asta C, Cirlini M, Morini E, Galaverna G (2011) Brand dependent volatile fingerprinting of Italian wines from Valpolicella. J Chrom A1218:7557-7565
20. Hirri A, DeLuca M, loele G, Balonki A, Bassbasi EM, Kzaiber F, Oussama A Ragno G (2015) Chemometric classification of citrus juices of Moroccan cultivars by infrared spectroscopy. Czech J Food Sci 33:137-142

21. Jordan MJ, Shaw PE, Goodner KL (2001) Volatile components in aqueous essence and fruit of Cucumis melo cv. fresh Athena (Muskmelon) by GC-MS and GC-O. J Agric Food Chem 49:5929-5933

22. Tikunov $Y$, Lommen $A$, Ric de Vos $C H$, Verhoeven $H A$, Bino RJ, Hall BR, Bovy AG (2005) A novel approach for non-targeted data analysis for metabolomics, large-scale profiling of tomato fruit volatiles. Plant Physiol 139:1125-1137

23. Farneti B, Khomenko I, Cappellin L, Ting V, Costa G, Biasioli F, Costa F (2015) Dynamic volatile organic compound finger printing of apple fruit during processing. LWT-Food Sci Technol 63:21-28

24. Farrell RR, Fahrentrapp J, Garcia-Gomez D, Sinues PML, Zenobi R (2017) Rapid finger-printing of grape volatile composition using secondary electrospray ionization orbitrap mass spectrometry: a preliminary study of grape ripening. Food Control 81:107-112

25. Steingass CB, Grauwet T, Carle R (2014) Influence of harvest maturity and fruit logistics on Pineapple (A. comosus L. Merr) volatiles assessed by headspace solid phase Micro extraction and gas chromatography-mass spectrometry (HS-SPME-GC-MS). Food Chem 150:382-391

26. Takeoka GR, Buttery RG, Teranishi R, Flath RA, Guentert M (1991) Identification of additional pineapple volatiles. J Agric Food Chem 39:1848-1851

27. Tokitomo Y, Steinhaus M, Buttner A, Schieberle P (2005) Odor-active constituents in fresh pineapple (Ananas comosus L. Merr) by quantitative and sensory evaluation. Biosci Biotechnol Biochem 69:1323-1330

28. Deshpanda AB, Chidley HG, Oak PS, Pujari KH, Giri AP, Gupta VS (2017) Isolation and characterization of 9-lipoxgenase and epoxide hydrolase 2 genes: Insight into lactone biosynthesis in mango fruit (Mangifera indica L.). Phytochem 138:65-75

29. ISO (2007) Sensory analysis. General guidance for the design of test room. HIS, Geneva

30. Schwab W (2013) Natural-4-hydroxy-2,5-dimethyl-3(2H)-furanone-A review. Molecules 18:6936-6951

31. Scheidig C, Czerny M, Schieberle P (2007) Changes in key odorants of raw coffee beans during storage under define conditions. J Agric Food Chem 55:5768-5775

32. NIST Standard Reference Database Number 69, National Institute of Standards and Technology, Gaithersburg MD, 20899. http://doi. org/10.18434/T4M88Q, Accessed 22 Nov 2018

33. El-Sayed A (2005). Pherobase. HortResearch, Lincoln, New Zealand. http:// www.pherobase.com. http://www.pherobase.com/database/kovats/ kovats-index.php

34. Lasekan O, Ng SS (2015) Key volatile aroma compounds of three black velvet tamarind (Dialium) fruit species. Food Chem 168:561-565

35. Lasekan O (2017) Identification of the aroma compounds in Vitex doniana sweet: free nand bound odorants. Chem Cent J 11:19-25

36. Lasekan O, Khatib A, Juhari H, Patiram P, Lasekan S (2013) Headspace solid-phase micro extraction gas chromatography-mass spectrometry determination of volatile compounds in different varieties of African star apple fruit (Chrysophillum albidum). Food Chem 141:2089-2097

37. Schieberle P (1995) Recent developments in methods for analysis of flavour compounds and their precursors. In: Gaonkar A (ed) Characterization of food: emerging methods. Elsevier, Amsterdam, pp 403-431 